\title{
Mathematical enculturation from the students' perspective: shifts in problem-solving beliefs and behaviour during the bachelor programme
}

\author{
Jacob Perrenet • Ruurd Taconis
}

Published online: 18 November 2008

(C) The Author(s) 2008. This article is published with open access at Springerlink.com

\begin{abstract}
This study investigates the changes in mathematical problem-solving beliefs and behaviour of mathematics students during the years after entering university. Novice bachelor students fill in a questionnaire about their problem-solving beliefs and behaviour. At the end of their bachelor programme, as experienced bachelor students, they again fill in the questionnaire. As an educational exercise in academic reflection, they have to explain their individual shifts in beliefs, if any. Significant shifts for the group as a whole are reported, such as the growth of attention to metacognitive aspects in problem-solving or the growth of the belief that problem-solving is not only routine but has many productive aspects. On the one hand, the changes in beliefs and behaviour are mostly towards their teachers' beliefs and behaviour, which were measured using the same questionnaire. On the other hand, students show aspects of the development of an individual problem-solving style. The students explain the shifts mainly by the specific nature of the mathematics problems encountered at university compared to secondary school mathematics problems. This study was carried out in the theoretical framework of learning as enculturation. Apparently, secondary mathematics education does not quite succeed in showing an authentic image of the culture of mathematics concerning problem-solving. This aspect partly explains the low number of students choosing to study mathematics.
\end{abstract}

Keywords Mathematical problem-solving - Behaviour - Beliefs · Enculturation · Higher education · Secondary education

\section{Introduction}

Science and mathematics education fall behind in fostering talent and stimulating young people seeking a career in these domains (Organisation for Economic Co-operation and Development 2006). This problem involves low popularity as well as (perceived) difficulty

J. Perrenet $(\triangle) \cdot R$. Taconis

Eindhoven School of Education, Eindhoven University of Technology, P.O. Box 513,

5600 MB Eindhoven, the Netherlands

e-mail: j.c.perrenet@tue.nl 
(National Research Council 2001; Hoyles, Newman \& Noss 2001). It is feared that educational practice is a limiting factor in the development of a science-oriented, knowledge-based economy as is desired by the European Council in the Lisbon Treaty (European Council 2000). A main issue is the discontinuity between secondary education and university education. Although discontinuities on the cognitive level concerning both skills and conceptual understanding between secondary school and university play a role in this phenomenon, our theoretical perspective for looking at these problems will be a cultural one, using the concept of enculturation. In the next sections, we shall clarify the theoretical perspective of enculturation and discuss some research results from science and mathematics education. After that, we shall describe our study in the domain of mathematics education, focussing on mathematical problem-solving and focussing on the students that successfully choose a bachelor career path in mathematics. By looking at the characteristics of their enculturation process, their successful transition from a school mathematics culture to a university mathematics culture, we hope to find reasons why other students did not choose mathematics or were not successful.

\section{Enculturation}

\subsection{Theoretical perspective}

Following a socially oriented perspective on learning (Vygotsky 1978; Lave \& Wenger 1991; Wenger 1999), science and mathematics education can be seen as a process of enculturation of learners into the culture of science and mathematics. We define enculturation as a process of voluntary participation and gradual commitment in which the learner's identity is enriched by incorporating sub-cultural elements so as to facilitate participation progressively (Taconis, in preparation). But also, enculturation is a product of participation in authentic activities (Wenger 1999).

The learning of a subject's cognitive content is considered a process embedded within the more comprehensive process of enculturation which provides the cultural prerequisites necessary for learning. Becoming a mathematician or a scientist involves enculturation. Krogh and Thomsen (2005) have shown how the number of intercultural borders between a learner's initial position and the science culture one is to enculture in correlates significantly with avoiding, for instance, A-level physics.

The enculturation path that education should pave for the students is often bumpy or broken. A particular bump is the transition from secondary education to higher education. Students from secondary education may lack some of the knowledge or skills needed for success in higher education. Moreover, they may have been trained in a too schoolish manner with schoolish problem-solving assignments alone that imply a false type of enculturation connected with a false schoolish image of the subject (Civil \& Andrade 2002).

So, using the enculturation perspective, we can interpret the problems in a new way. This explanation of the problems, as well as its directions for solutions is called the enculturation hypothesis in this paper.

\subsection{Enculturation into mathematics and mathematical problem-solving}

Within the context of mathematics education, the term enculturation means enculturation into mathematics. In this way it is used by Schoenfeld (1992). The main point of the 
enculturation hypothesis in this sense-as explained in Section 2.1-is that the majority of secondary school students are hindered in their mathematical career because of the culture of school mathematics, as it differs significantly from the professional culture of mathematics. According to FitzSimons (1999), school presents a picture of mathematics and its cultural values that does not match the academic reality. According to Skovsmose and Nielsen (1996), values and meanings which are not explicitly expressed by teachers can nevertheless be inferred from their actions and interactions with the students. Thus, inferences drawn by students of their teacher's mathematical expectations can deter them from engaging in mathematics. This incorrect picture of mathematics as a discipline can hinder even gifted students in developing a positive relationship - as it is called by Boaler (2002a, b) -with (their own) mathematical knowledge.

Ratner (2000) describes cultures using a list of the following elements: values, artefacts, activities, concepts, goals, perceptions and beliefs. The element of activities appears to be the most influential. Enculturation into mathematics comprises not only the cautious adoption of these specific elements of the mathematical culture. Some cultural elements seem particularly important for the enculturation into mathematics, either due to their prominent role within mathematics or due to the problems students encounter in integrating them. According to Halmos, mathematics consists of axioms, theorems, proofs, definitions, theories, formulas and methods - and, to include applied mathematics, one should add models to Halmos' list - but what mathematics really consists of is problems and solutions (Halmos 1980). From Halmos' and Ratner's perspective, the activity of mathematical problem-solving can be considered the most important element of the culture of mathematics.

In the present study, we have chosen to investigate mathematical problem-solving beliefs and behaviour mainly from the students' perspective. A system of beliefs-within the context of mathematics - is defined by Schoenfeld (1985, p. 5) as "the set of understandings about mathematics that establish the psychological context within which individuals do mathematics". We will look at students' beliefs about technical, metacognitive and productive aspects of mathematical problem-solving and at their reports about their own behaviour regarding these aspects, staying close to the framework of Schoenfeld (1985). The term problems can refer to small problems (tasks) as well as large problems (assignments); we use the term in this broad sense.

We are aware that there is not just one monolithic mathematical culture. Ernest (1991) distinguishes as many as five mathematics education sub-cultures - the industrial trainers, the technological pragmatists, the old humanists, the progressive educators and their public educators - with differing features concerning their view of mathematics, their theory of learning and assessment, their theory of teaching mathematics, mathematical problemsolving, etc. Also, various professional mathematical sub-cultures do exist, such as pure mathematics, applied mathematics and sub-cultures within these two. Our main focus is not the differences between the various educational or professional sub-cultures, but the differences between common aspects of the contemporary school mathematics culture and the university mathematics culture, especially concerning the central element of mathematical problem-solving.

\subsection{Our questions about enculturation during the bachelor programme}

Given the fact that the school mathematics culture is different from the professional university mathematics culture, we can suppose that the bachelors students' mathematics 
culture will be somewhere in between. Our research questions concerning the problemsolving aspect of enculturation into mathematics are the following:

- Do students' mathematical problem-solving beliefs and behaviour change during their bachelor years?

And if so,

- Do they change towards the professional mathematical problem-solving beliefs and behaviour?

And if so,

- Which factor(s) cause these changes, according to the students?

\section{Method}

\subsection{Type of study}

Our research into the students' problem-solving beliefs and behaviour can be characterised as a field study with a pre-experimental one-group, pre-test post-test design. The post-test was accompanied by a retrospective self-report about individual substantial changes in beliefs and behaviour. The research into the professionals' problem-solving beliefs and behaviour is a one-shot case study; it is only used as a benchmark. The details concerning subjects, context and tools are explained in the following sections.

\subsection{Subjects and the context of data collection}

At the Technische Universiteit Eindhoven, the number of students completing their bachelor's degree in Applied Mathematics each year is (only) about ten. In order to collect enough data, we followed five consecutive cohorts (from 2000 and onwards). A questionnaire about problem-solving beliefs was presented twice. The first time was on the students' first day at the university. The second time - only for those students who were still in the programme - was at the end of one of the final bachelor courses: a course about academic reflection in the context of mathematics (explained below).

The curriculum partly reflects the staff's research activities which characterise the local mathematical culture. These activities are aimed at improving existing mathematical methods as well as developing new methods for technical sciences and industry. Mathematical areas are analysis (analytical as well as numerical), scientific computing, operations research, statistics and discrete mathematics. The curriculum includes a series of mathematical modelling projects in which pairs of students solve open mathematical problems posed in non-mathematical language. We give three examples (Perrenet \& Adan 2002).

On a machine in a sweet factory, marshmallows are sealed in fixed batches. The marshmallows are transported to this machine on a conveyor belt. The arrival process is irregular, i.e., the inter-arrival times of the marshmallows vary. However, the machine works continuously at constant speed. To prevent the machine from sealing too many empty positions, a vertical buffer is positioned between the conveyor belt and the sealing machine. Develop a model to determine the optimal buffer size. 
Teletext is an on-line information medium available on television. It usually takes some time before a selected information page appears on the screen. Develop a model to explain the delay, and, if possible, propose a method to reduce the delay.

For the design of a new airplane one has to take into account international regulations for the production of noise. In these regulations it is stated that the amount of noise produced by an airplane landing or taking off should not exceed certain thresholds. Most of the noise produced by airplanes comes from their engines; their location within the aircraft is possibly a significant factor in the noise production: above the wings at the tail, or below them. The claim is that in the first case the wings yield a considerable reduction of noise. Develop a model to estimate the reduction of noise.

Students have to find out for themselves which mathematical methods are applicable; often, various approaches are possible and sometimes the best approach has not yet been taught to them. The students build a portfolio during the process and, in a concluding course, they reflect on their former modelling activities from various perspectives. As an example, they have to look back at their earlier projects and construct plans for improvement, given the extra knowledge and skills they have meanwhile accumulated. Another assignment, especially relevant for this investigation, consists of answering the questions of the questionnaire about problem-solving beliefs and behaviour for the second time, referring back to their individual results of the first time and comparing those with the results of the second time, and — in the form of an individual assignment — giving a written individual explanation for their own possible substantial differences. The assignment's exact wording is given in Section 3.3. See publications by Perrenet and Adan (2002) and Perrenet and ter Morsche (2004) for more details about the educational context.

The scientific staff of the Mathematics Department was asked to participate voluntarily in the investigation by filling in the questionnaire about their own mathematical problemsolving beliefs and behaviour, in this way giving a picture of the local $^{1}$ mathematical problem-solving culture.

\subsection{Tools}

Starting from a sub-scale of an existing questionnaire (Pelgrum, Eggen, Plomp, \& Kuper 1984) on thinking mathematically, items were selected and adapted in discussion with some members of the department. Extra items were inspired by ideas presented by Schoenfeld (1985) and Ernest (1991). In this selection, three concepts in particular-indicated in literature as key components of emerging mathematical identities - were kept in mind: metacognitive thinking, perception of mathematics in the context of mathematical problem-solving and technical approach to mathematical problems. The items are fully verbalised (translated from Dutch) in Table $1^{2}$. They were answered with a Likert scale $(1=$ totally disagree to $5=$ totally agree). In the last column, we give the abbreviations to be used in the Appendix.

The reflection assignment was worded as follows:

Study the completed questionnaire from the start of your first year and the one just completed. Report both lists of results. Make a note of substantial differences (with a

\footnotetext{
${ }^{1}$ Of course, the professional mathematical culture in general has much more variety than the university culture alone, and also the technical university culture, the culture of applied mathematics, has specific characteristics.

${ }^{2}$ In fact, the list the students used for reflection was longer. Only the items with relevance for enculturation are included here.
} 
Table 1 Items of questionnaire on mathematical problem-solving behaviour and beliefs

Fully verbalised

1. With a mathematical assignment I always start by very precisely reading through the givens and what is asked for.

2. When I do not succeed in solving a mathematics problem, I often try to make a Sketch, drawing, table sketch, a drawing or a table.

3. Whilst solving a mathematics problem I regularly ask myself whether I am on On the right track the right track.

4. Sometimes I first try to estimate the outcome of a mathematics assignment. Estimating the answer

5. When I have found the answer of a mathematics assignment I check it, before I Checking the answer start with the next one.

6. Sometimes I try to investigate whether my own solution method of a mathematics assignment is the most convenient.

7. In mathematics it is often necessary to formulate in a precise way.

8. You should always give a very precise answer in a mathematical assignment: for example it is not allowed to round off the numbers in your answer.

9. In mathematics it is hardly necessary to learn something by heart.

10. Mathematics is $90 \%$ insight and $10 \%$ work.

11. Some mathematics assignments can be solved by using common sense, without using rules.

12. Most mathematical assignments can be solved in one way only.

13. Sometimes a mathematical property is so self-evident that proof is not necessary.

14. Mathematics as a subject is complete; truly new knowledge is not added.

15. One can invent new mathematics oneself.

16. The solution of a mathematical assignment is sometimes very unexpected.

17. Applied mathematics has available ready-made methods to solve mathematical problems from other technical disciplines.
Abbreviated

Reading precisely

Most convenient solution method

Formulating precisely

Always precise answer

Learning by heart not necessary $90 \%$ insight $10 \%$ work

Common sense

One way only

Proof not necessary

Subject complete

Inventing mathematics

oneself

Unexpected solution

Ready-made methods

shift of two at least) between both lists. Write an explanation for each substantial shift. What was the cause of the change? What happened that caused you to change substantially on a specific aspect?

\section{Results}

\subsection{Overview}

The students' retrospective self-reports (explanations of substantial individual shifts) were paraphrased and summarised. This summary was discussed and checked with students of the last cohort. Typical original explanations and the summary are discussed in Section 4.3 on qualitative analysis. The main objective of the tool was elicitation of this qualitative response to get the students' perspective. However, to give a global picture of the shifts first, the results of statistical analysis are presented in Section 4.2 at the level of the list as a whole as well as at item level with inclusion of the (quantitative) data of the professional mathematicians. In Section 4.4, we combine qualitative and quantitative analysis. 
Table 2 Students' means and experts' means

\begin{tabular}{|c|c|c|c|c|c|}
\hline Factor $^{\mathrm{a}}$ & $\alpha$ & $\begin{array}{l}\text { Bachelor students } \\
\text { novice }(N=47)\end{array}$ & $\begin{array}{l}\text { Bachelor students } \\
\text { experienced }(N=47)\end{array}$ & $\begin{array}{l}t \text { (novice- } \\
\text { experienced) }\end{array}$ & $\begin{array}{l}\text { Experts } \\
(N=24)\end{array}$ \\
\hline $\begin{array}{l}\text { 1. Precise and metacognitive } \\
\text { processes in mathematical } \\
\text { problem-solving (items }{ }^{\mathrm{b}} 3,4 \text {, } \\
5,6,7,13 \mathrm{i} \text { ) }\end{array}$ & 0.63 & 3.32 & 3.78 & $4.3 * * *$ & 4.37 \\
\hline $\begin{array}{l}\text { 2. Productive aspects of } \\
\text { mathematical problem- } \\
\text { solving (items } 12 \mathrm{i}, 14 \mathrm{i}, 15 \text {, } \\
16,17 \mathrm{i}\end{array}$ & 0.56 & 3.64 & 4.06 & $3.0 * *$ & 4.33 \\
\hline $\begin{array}{l}\text { 3. Technical approach to } \\
\text { mathematical problem-solving } \\
\text { (items } 1,2,8,9,10,11 \text { ) }\end{array}$ & 0.42 & 3.51 & 3.54 & 0.241 & 3.53 \\
\hline
\end{tabular}

\subsection{Quantitative analysis of shifts}

Explorative factor analysis showed three factors emerging from the data that indeed roughly represented the three concepts that guided the instrument construction (Table 2).

The first factor roughly denotes precise and metacognitive processes in mathematical problem-solving. An analysis on item level showed that all variables included in the factor contribute to this result since they all shift in this direction. The presence of items 7 (in mathematics, it is often necessary to formulate in a precise way) and $13^{\mathrm{i}}$ (sometimes a mathematical property is so self-evident that proof is not necessary: inverted) was unexpected, but seems to fit in the scale quite well. ${ }^{3}$ Students clearly shift towards the expert view (Table 2, last column) and put more emphasis on a precise and metacognitive approach to mathematical problems.

The second factor denotes productive aspects of mathematical problem-solving. The students' belief concerning this aspect of problem-solving significantly shifts in the direction of the experts' belief.

The last factor, technical approach to mathematical problem-solving, shows a low value for the homogeneity index Cronbach's alpha. Splitting the scale into two more homogenous parts, problem analysis (items 1,2 and 8) and view on the demands of mathematical problem-solving (items 9, 10 and 11), does not solve the problem. Apparently, the various skills and views on mathematical tasks are like problem-solving styles, individually different approaches/views, but neither of these is particularly more mathematical than the other. No significant shift has been found for this scale, but due to this measuring problem, the result cannot be interpreted. A more detailed analysis is made at the level of individual items.

In Table 3, for every item, the means and standard deviations for the first and second students' measurement as well as for the experts' measurement are presented. For every item, it is indicated when the student difference is significant. Ten out of 17 items show a significant shift of the students' position towards the experts' position, four out of 17 items

\footnotetext{
${ }^{3}$ Leaving these out, the scale formed by items 3, 4, 5 and 6 shows an alpha of 0.72 .
} 
Table 3 Means and standard deviations per item for students and for experts

\begin{tabular}{|c|c|c|c|c|c|c|}
\hline \multirow[b]{2}{*}{ Item } & \multicolumn{2}{|c|}{$\begin{array}{l}\text { Bachelor students } \\
\text { novice } N=47\end{array}$} & \multicolumn{2}{|c|}{$\begin{array}{l}\text { Bachelor students } \\
\text { experienced } N=47\end{array}$} & \multicolumn{2}{|c|}{$\begin{array}{l}\text { Expert } \\
\text { mathematicians } \\
N=26\end{array}$} \\
\hline & Mean & SD & Mean & $\mathrm{SD}$ & Mean & SD \\
\hline 1. Reading precisely*** & 3.83 & 0.99 & 4.32 & 0.69 & 4.77 & 0.51 \\
\hline 2. Sketch, drawing, table** & 3.70 & 0.98 & 4.13 & 0.74 & 4.40 & 0.71 \\
\hline 3. On the right track $* * *$ & 3.06 & 1.22 & 3.85 & 0.83 & 4.23 & 0.82 \\
\hline 4. Estimating the answer* & 3.17 & 1.20 & 3.55 & 1.02 & 4.24 & 0.72 \\
\hline 5. Checking the answer & 3.43 & 1.02 & 3.62 & 1.05 & 4.35 & 0.89 \\
\hline 6. Most convenient solution method $* * *$ & 2.72 & 1.14 & 3.53 & 1.02 & 4.44 & 0.77 \\
\hline 7. Formulating precisely** & 4.26 & 0.77 & 4.66 & 0.52 & 4.92 & 0.28 \\
\hline 8. Always precise answer* & 3.85 & 1.18 & 3.43 & 1.46 & 3.36 & 1.22 \\
\hline 9. Learning by heart not necessary & 2.85 & 1.16 & 3.04 & 1.02 & 2.81 & 1.30 \\
\hline 10. $90 \%$ insight, $10 \%$ work $* * *$ & 3.23 & 1.16 & 2.57 & 1.10 & 2.16 & 0.80 \\
\hline 11. Common sense & 3.62 & 1.09 & 3.72 & 0.99 & 3.60 & 1.32 \\
\hline 12. One way only & 1.64 & 0.85 & 1.45 & 0.75 & 1.58 & 0.95 \\
\hline 13. Proof not necessary & 2.74 & 1.22 & 2.55 & 1.25 & 2.08 & 1.13 \\
\hline 14. Subject complete ${ }^{* * *}$ & 2.02 & 1.13 & 1.32 & 0.63 & 1.19 & 0.40 \\
\hline 15. Inventing mathematics oneself*** & 3.15 & 1.25 & 3.85 & 1.01 & 4.71 & 0.46 \\
\hline 16. Unexpected solution & 3.89 & 0.98 & 4.13 & 0.82 & 4.50 & 0.71 \\
\hline 17. Ready-made methods & 3.17 & 1.01 & 2.89 & 1.03 & 2.77 & 1.37 \\
\hline
\end{tabular}

As novice and as experienced bachelor

$* p<0.05, * * p<0.01, * * * p<0.001$; significance of the difference between novices' mean and experienced students' mean (one-tailed, paired samples $t$ test)

show a non-significant shift (a trend) in that direction and no items show a significant shift in the opposite direction.

Inspection of the distribution of the variables indicated that, for items 1, 7, 12, 14 and 15 , the normality of the distribution was not evident. Therefore, the results for these items, found using the $t$ test, were verified using the Wilcoxon signed ranks non-parametric test. This analysis fully supported the results on the variables shown in Table 3.

For most items, the standard deviation gets smaller through the years (Table 3, third and fifth columns) and is even smaller for the staff (seventh column). For some items, however, the standard deviation is quite large for the staff's responses on beliefs and behaviour. We come back at this result in Section 4.4.

\subsection{Qualitative analysis of shifts}

A substantial shift was defined as a difference of at least two points on the scale. ${ }^{4}$ Students gave their explanations of possible individual substantial shifts. We give two examples.

John, about his shift on item 3 (on the right track; shift from 2 to 4 ):

At the start of the first year, I indicated 2 for this proposition, I moderately disagreed. Now, I indicate 4, as I moderately agree now. The main cause is that the mathematics problems I met at school came up within the context of a certain technique (such as a

\footnotetext{
${ }^{4}$ In hindsight, this is - for almost every item - comparable with two standard deviations of the distribution of differences.
} 
chapter with only problems about integration) or they were solved using standard recipes (such as "Investigate the function and draw the graph."). For these kinds of problems the approach to be followed was so clear that the first try in almost all cases directly led to the solution. At university it quickly became clear that problems often consist of several sub-problems and one has to choose from several available techniques, some of which get hopelessly stuck.

Peter, about his shift on item 14 (subject complete; shift from 3 to 1):

When I started at university, I just possessed school mathematics and nothing more. In my opinion then, not much expansion could be thought of. So I thought that mathematics as a discipline was complete. However, now it has appeared that, in mathematics, a lot of things have to be investigated and discovered. Very often in lectures you hear about domains with open problems, unproved postulates and interesting stuff to be improved. So my opinion now is that mathematics is far from complete, as much can be discovered and added. Every new mathematical discovery gives way to more and deeper investigations. Mathematics is a discipline that does not stand still; new knowledge is always added.

The first example concerns item 3 (Whilst solving a mathematics problem I regularly ask myself whether I am on the right track). The quantitative analysis pointed out that this item is part of the factor precise and metacognitive processes in mathematical problem-solving (Table 3). John's explanation of his substantial shift in behaviour points to the difference in character of the mathematics problems met at secondary school and the problems met at university. The complexity of the university problems makes another kind of behaviour necessary.

The second example concerns item 14 (Mathematics as a subject is complete; truly new knowledge is not added), part of the factor productive aspects of mathematical problemsolving (Table 3). Peter's explanation points to the fact that only at university he heard about the existence of open problems and things to be proved and improved. This experience has changed his belief about the domain in the direction of it being an incomplete and productive discipline.

In the Appendix, the explanations for the substantial shifts on all items are paraphrased and summarised. The number of substantial shifts per item varies from four to 19 (out of 47 students); the numbers are indicated in the Appendix. On average per item, more than $20 \%$ of the students show a substantial shift. For some items, individual substantial shifts are only in one direction (towards the experts' mean). For most items, there are substantial individual shifts in both directions (towards as well as away from the experts' mean); for every item, most substantial individual shifts are towards the experts' mean. For every item, the explanations in the same direction appeared to be more or less similar. For each item and direction, the contents of the explanations could, therefore, be characterised by a few lines (see the Appendix). We interpret these explanations to get more insight into the causes of change in belief and behaviour from the students' perspective.

\subsection{Quantitative and qualitative analysis combined}

In Table 2, the items are indicated that belong to the two factors that show a significant shift (precise and metacognitive processes in mathematical problem-solving and productive aspects of mathematical problem-solving). We assume that the explanations of the individual students with substantial shifts for these items are also relevant for the 
significant shifts of the group as a whole. As expected, for these items, only a small minority of the substantial shifts are away from the experts. In Table 4, the explanations for the various individual substantial shifts towards the experts are summarised for those two factors. The overall picture emerging from the students' explanations, underlying the two factors with significant shifts, is the following (see Table 4).

Precise and metacognitive processes in mathematical problem-solving According to the students, the mathematical problems met at university are quite different from the problems met at school. Problems at secondary school were standard and, almost always, there was a unique and easy method for solving a problem: just applying the right rules. Hence, estimating the answer, checking whether one is on the right track, checking an answer afterwards and asking oneself whether the solution method used was the most convenient were not really necessary. Problems at university, on the other hand, were perceived as much more complex and non-standard. Therefore, at university, it is more necessary and more useful to perform the kind of problem-solving activities mentioned in the foregoing. Also, formulating precisely is more important: it prevents mistakes and is necessary for communication. School mathematics was simply the application of rules; at university, a totally new argument is sometimes needed to solve a problem. Therefore, university mathematics is much more interesting and exciting. Providing proof is always necessary or useful.

Productive aspects of mathematical problem-solving According to the students, the view of mathematics one gets at secondary school is that of a basically static body of knowledge. The experience of mathematics at university has shifted the students' view towards the perspective that, in mathematics, many unsolved problems and unproved methods are waiting for them. Also, when a solution or proof has been found, a new problem will arise. Mathematics solutions to university problems can be surprising. To apply mathematics to other fields, further development of methods is called for.

Some items of the third, non-significant, factor technical approach to mathematical problem-solving show a significant shift at item level (Table 3). In Table 4, the explanations of the directional shifts towards the experts belonging to these items are summarised. Again, we assume that the explanations of the individual students with substantial shifts for these items are also relevant for the significant shifts of the group as a whole.

Technical approach to mathematical problem-solving The overall picture emerging from the students' explanations in Table 4, belonging to those items of the third factor, which are significant at item level, is the following: According to the students, at school, activities at the start of solving a mathematics problem, such as reading precisely or making a sketch or a table, were not necessary. At university, problems are non-standard; precisely reading the question is important. To gain insight into a problem and its solution, activities such as making a sketch or a table are useful. At school, precise answers were possible. For problems encountered at university, precise answers are often not possible and approximations are good enough; the solution method is more important than the answer. At school, insight into mathematics came easily to these students, and hard work was not necessary; at university hard work is sometimes necessary, although the work in itself is not always difficult; on the other hand, much practice (hard work) is now necessary to acquire insight.

The main factor for their change in belief and behaviour, as reported by the students, is clearly the experience with authentic mathematical problems. 
Table 4 Characterisation of explanations of substantial shifts towards the experts

Item

Explanations for factor precise and metacognitive processes in mathematical problem-solving

3. On the right track At school I was rather sure of myself; I knew I was on the right track. Problems were given in the context of a certain technique or they asked for a standard recipe (like "Investigate the function and draw the graph."). At school I never had the experience of being on the wrong track. What's the use of asking oneself whether one is on the right track when there is only one track, like with school mathematics? I could use my solving skills blindfolded. Now the problems are bigger and more complex. Now it saves time to halt now and then and ask oneself whether one is on the right track.

4. Estimating the answer At school my answers were right most of the time, so estimating was not necessary. We did not learn a systematic method for estimation; we did not know the background of problem-solving methods. I could not do it by heart. At university it is useful and we know how to do it. I estimate because I value intuition.

5. Checking the answer At school I worked by reading/thinking/writing down the solution/next one; now I look at the answer to see whether it makes sense or I perform a direct control (for instance in a differential equation). Whenever possible, I do check an answer.

6. Most convenient solution method

At school most of the times only one method existed; problems were right in one try; solutions were always short; Now there is a choice of methods and some of those take lots more time than others. Now it is more interesting to look back.

7. Formulating precisely At school I was satisfied when I understood what I meant myself. Now I have a different opinion. Formulating in a precise way makes your mathematics more understandable for others, it prevents mistakes, and it is nicer to look at.

13. Proof not necessary However self-evident a property, the proof can still be difficult; proving gives you insight into the situation. Some properties look trivial for real numbers, but they are not for other structures.

Explanations for factor productive aspects of mathematical problem-solving

12. One way only

14. Subject complete

15. Inventing mathematics one self

16. Unexpected solution

17. Ready-made methods

Explanations for factor technical approach to mathematical problem-solving ${ }^{\mathrm{a}}$

1. Reading precisely makes mathematics exciting.
I have a broader view of problems; often various perspectives exist.

I never knew that mathematics is more than calculation only. When you have had mathematics at secondary school, you think that is all there is. Now I know there are lots of unsolved problems and unproved methods. And when a solution or proof has been found, a new problem will arise.

At the start of my studies I though mathematics was a more-or-less stable and complete body of knowledge. I have realised now that some theorems are only a few years old. Some of our teachers invent new mathematics. I have done a project in relatively unknown territory. There is a lot of work waiting for us.

At school I was convinced that by thinking and estimating you could be quite sure about the kind of solutions problems would bring about. Nowadays I have often seen surprising answers. At school it was simply the application of rules; now a totally new argument can be needed for a solution; that is what

There are standard methods, but for every case a method has to be tailored to fit the concrete problem; also it is often necessary to create a convenient mathematical model. Mathematics doesn't have all the answers; there are a few standard methods that can be used, but the rest is theory-based improvisation.

Assignments in secondary education were standard; their structure was often the same. To solve them, it was enough to see the relevant figures and 
Table 4 (continued)

Item

functions. At university assignments are not standard. The problem statement
gives information about what is allowed and what is not. Now, you sometimes
have to read the question again and again before you understand what is
actually asked for. With mathematical modelling, one encounters all sorts of
boundary conditions and nuances; so reading the question is almost more
important than finding the solution.
At school a sketch or drawing was not necessary in most cases; it only took
time. You could easily imagine the problem situation. Now it is useful, not
only for solving the problem but for gaining insight into the problem and the
solution. For instance with threefold integrals in order to delimit the
boundaries and with proof to check whether a theorem is true or not.
Now a good approximation is often good enough. There are many problems
where a precise answer is not possible. The experience with numerical
analysis and with simulations has taught me that an exact answer is not always
necessary. At school the problems were of a type where a precise answer was
possible. In the real world a precise answer is almost impossible. And besides
that, it is more the way to the answer that is more important than the answer
itself. I am not against rounding off in general; I am against rounding off too
early. Now I think rounding off is less ugly than I thought at school.

${ }^{a}$ Only for items with a significant shift at item level

Explanations for shifts away from the professionals' mean Finally, we explore the phenomenon that individual substantial shifts not only occur in the direction towards the professionals' mean, but sometimes away from it (see the Appendix). For most items, the number of substantial shifts away from the professionals is negligible. This is not the case for items 8, 9, 11, 12, 13 and 17. However, Table 3 shows, for almost all of these items, ${ }^{5}$ a large standard deviation of the professional mathematicians' responses, namely, a standard deviation of about 1 or greater than 1 . In fact, this result suggests a variation of underlying belief and behaviour aspects in the direction of various sub-cultures. Variation could be expected because (1) the department performs fundamental as well as applied research, (2) there are beginning researchers as well as researchers at the end of their career and (3) there are researchers who studied at the university where they are employed now as well as researchers from other universities, even from other countries. So, most of the times, a substantial shift away from the professionals' mean can be interpreted as a shift towards an acceptable position in a broad range. This can be interpreted as the development of aspects of an individual professional style. The

\footnotetext{
${ }^{5}$ Item 8 excluded.
} 
acceptability of different positions in these cases can be illustrated by the characterisation of the explanations given in the Appendix.

8. (Always precise answer) Appreciation of approximated answers within a numerical analysis and simulations approach (modelling) versus appreciation of precise answers within a fundamentalistic approach;

9. (Learning by heart not necessary) Knowing standard formulas by heart for efficient use versus knowing the relations between formulas so deduction is possible;

11. (Common sense) Seeing results through intuition shaped by experience versus reaching results step-wise using rules;

12. (One way only) Using multiple methods from various perspectives or recognising structural analogy in different methods;

13. (Proof not necessary) Proving every detail in a proof (formalistic) versus sketching a convincing outline of a proof (communicative);

17. (Ready-made methods) Tailoring standard methods versus recognising structural analogies of application methods.

\section{Conclusion and discussion}

\subsection{Conclusions}

Mathematical problem-solving is a central element in the culture of mathematics. During their bachelor years, according to their own reports, mathematics students change their mathematical problem-solving beliefs and behaviour. For experienced bachelor's degree students, the precise and metacognitive processes and the productive aspects of mathematical problem-solving are more important than when they were novice students. During mathematical problem-solving, experienced bachelor's degree students, more often than when they were novices, are involved in estimating the answer beforehand, formulating precisely, asking themselves whether they are on the right track and investigating afterwards whether the solution method used is the most convenient solution method. As experienced bachelor's degree students, they believe more often that mathematics is an open and growing body of knowledge, as it is seen less as a complete subject, and they believe more often that one can invent mathematics oneself. In their technical approach to mathematical problem-solving, behaviour such as reading precisely at the start of solving a problem and making a sketch or a table has become more important. On the other hand, giving a precise answer has become less important and mathematics is no longer "mainly insight and little work".

On average, the students' shifts in beliefs and behaviour are towards the beliefs and behaviour of the professionals (their teachers). Some individual substantial shifts are away from the professionals' mean. However, most of the time, this can be interpreted as shifting towards a professional position within a broad range because of the large variation in the professionals' responses in these cases and because of acceptable qualitative explanations.

When students explain their own shifts in beliefs and behaviour, they mainly point to differences between mathematical problem-solving at secondary school and at the university. According to them, these differences are caused by the difference in nature of mathematics problems at university and at school. School problems are more closed, standard and easy; university problems are more open, challenging and complex. No student referred to reading about mathematics when explaining shifts. The students mainly referred to their problem-solving activities. 
Our conclusions in terms of enculturation are twofold. Apparently (Dutch) secondary school instills a kind of mathematical beliefs and behaviour in students that does not align well with the professional mathematical beliefs and behaviour. University education does, indeed, succeed in changing beliefs and behaviour towards a more professional position, especially through participation in more authentic activities.

\subsection{Discussion and implications for teaching}

Our study produced some clear results. However, we did not derive them from quantitative analysis alone. For that, the questionnaire needs further development. Especially the third factor, technical approach to mathematical problem-solving, did appear to be nonhomogeneous. The qualitative data of students' individual reflections about their shifts in beliefs and behaviour provide enough support for the main conclusions.

As for the professional mathematicians, we should realise that the quantitative results showed only a glimpse of their problem-solving beliefs and behaviour as part of their culture. The results indicated that, in some aspects, the variation was too large to speak of one uniform culture; probably one should speak of aspects of sub-cultures. Also, we should realise that this professional culture is a culture of applied mathematicians. We believe that we can generalise the main results to mathematicians in general (including the culture of pure mathematics), but not for all details. For example, the results about the necessity of always giving a precise answer are probably typical for applied mathematics. Also, the fact that the Eindhoven mathematics curriculum gives the students the opportunity to experience working at complex and open problems (modelling projects) for longer times is important for the outcomes.

The main point for improvement of secondary mathematics education for the potential mathematics students would be to give this group extra, more challenging mathematics problems.

Acknowledgement We thank the students and staff of the Mathematics Department for their cooperation. We thank the programme director of the Bachelor of Applied Mathematics, Hennie ter Morsche, for his constructive remarks on an earlier version of this article.

Open Access This article is distributed under the terms of the Creative Commons Attribution Noncommercial License which permits any noncommercial use, distribution, and reproduction in any medium, provided the original author(s) and source are credited.

\section{Appendix}

Table 5 Paraphrased and summarised students' explanations of substantial shifts with the number of substantial shifts indicated per item (out of 47 students)
Item
Explanations of shift towards and away from professionals (if any)

1. Reading precisely $N=6$

Shift towards professionals $(N=6)$ : Assignments in secondary education were standard; their structure was often the same. To solve them, it was enough to see the relevant figures and functions. At university assignments are not standard. The problem statement gives information about what action is allowed and what is not. Now, you sometimes have to read the question again and again before you understand what is actually asked for. With mathematical modelling, one encounters all sorts of boundary conditions and nuances; so reading the question is almost more important than finding the solution. 
Table 5 (continued)

2. Sketch, drawing, table $N=7$ Shift towards professionals $(N=6)$ : At school a sketch or drawing was not necessary in most cases; it only took time. You could imagine the problem situation easily. Now it is useful, not only for solving the problem but for getting insight into the problem and the solution. For instance with threefold integrals in order to delimit the boundaries, and with proof to check whether a theorem is true or not.

Shift away from professionals $(N=1)$ : The problems at school were better suited for making a sketch etc., whereas now this is only needed in some courses like set theory, linear algebra and linear analysis.

3. On the right track $N=10 \quad$ Shift towards professionals $(N=10)$ : At school I was rather sure of myself; I knew I was on the right track. Problems were given in the context of a certain technique or they asked for a standard recipe (like "Investigate the function and draw the graph.”). At school I never had the experience of being on the wrong track. What's the use of asking oneself whether one is on the right track when there is only one track, like with school mathematics? I could use my solving skills blindfolded. Now the problems are bigger and more complex. Now it saves time to halt now and then and to ask oneself whether one is on the right track.

4. Estimating the answer $N=11$ Shift towards professionals $(N=9)$ : At school my answers were right most of the time, so estimating was not necessary. We did not learn a systematic method for estimation; we did not know the background of problemsolving methods. I could not do it by heart. At university it is useful and we know how to do it. I estimate because I value intuition.

Shift away from professionals $(N=2)$ : Lots of problems now have nonnumerical answers, so estimation is not really possible like at school.

5. Checking the answer $N=13$ Shift towards professionals $(N=12)$ : At school I worked by reading/thinking/ writing down the solution/next one; now I look at the answer to check whether it makes sense or I perform a direct control (for instance in a differential equation). When it is possible, I do check an answer.

Shift away from professionals $(N=1)$ : Now, I check regularly during solving, so at the end is not necessary any more. Lots of problems are about proving and then you check during the process, not at the end.

6. Most convenient solution Shift towards professionals $(N=17)$ : At school most of the times only one method $N=17$ method existed; problems were right in one try; solutions were always short; Now there is a choice of methods and some of those take lots more time than others. Now it is more interesting to look back.

7. Formulating precisely $N=4$ Shift towards professionals $(N=4)$ : At school I was satisfied when I understood what I meant myself. Now I have a different opinion. Formulating in a precise way makes your mathematics more understandable for others, it prevents mistakes, it is nicer to look at.

8. Always precise answer $N=14$ Shift towards professionals $(N=12)$ : Now a good approximation is often good enough. There are many problems where a precise answer is not possible. The experience with numerical analysis and with simulations has taught me that an exact answer is not always necessary. At school the problems were of a type that a precise answer was possible. In the real world a precise answer is almost impossible. And besides that, it is more the way to the answer that is more important than the answer itself. I am not against rounding off in general; I am against rounding of too early. Now I think rounding off is less ugly than I thought at school.

Shift away from professionals $(N=2)$ : I have become a strong opponent of rounding off. At school this was not very important. Now I know that 
Table 5 (continued)

Item

Explanations of shift towards and away from professionals (if any)

9. Learning by heart not necessary $N=19$

10. $90 \%$ insight, $10 \%$ work $N=19$

11. Common sense $N=7$

12. One way only $N=5$

14. Subject complete $N=10$

15. Inventing mathematics oneself $N=6$ rounding off can change your answer. I am even against rounding off nice figures like $p i$ and $e$.

Shift towards professionals $(N=9)$ : Now you have to learn a number of standard formulas and solution methods to master the ideas behind an application. Of course one can deduce those but it takes time. At school it was not necessary because we could use the formula sheet. Now we have to do without.

Shift away from professionals $(N=10)$ : At school I learned more by heart. Now I understand the relations between formulas and theorems, so learning by heart is not necessary as I can deduce them. At school I learned the things on the formula sheet by heart because it made application easier.

Shift towards professionals $(N=18)$ : At school insight was enough and easily acquired. Now we often have problems (for instance certain kinds of proof), which are not really difficult but they need a lot of work. Insight is still very important, but to get to insight you have to practise a lot.

Shift away from professionals $(N=1)$ : It is no longer the work involved with the problem that is important, but the insight into its structure. At school, to gain insight into the problems took no time, the problems were that easy; most of the time was in the solution process. Now most of the time goes into the analysis of the problem to gain insight.

Shift towards professionals $(N=3)$ : Because of practice and experience I can often see the result without calculation.

Shift away from professionals $(N=4)$ : One always uses rules, even when they are trivial, and everything has to be proved.

Shift towards professionals $(N=3)$ : I have a broader view of problems; often various perspectives exist.

Shift away from professionals $(N=2)$ : Often different ways of solving a problem are not really different essentially. Sometimes one method is much better than another one, so there really is only one suitable method.

13. Proof not necessary $N=12$ Shift towards professionals $(N=8)$ : However self-evident a property may be, the proof can still be difficult; proving gives you insight into the situation. Some properties look trivial for real numbers, but they are not for other structures.

Shift away from professionals $(N=4)$ : When proof becomes a tautology (for instance only by reformulation), then the proof can be omitted. All mathematics has to be proved by someone, but not again and again. You do not need to prove little things because if you do you slow down too much.

Shift towards professionals $(N=9)$ : I never knew that mathematics is more than calculation only. When you have had mathematics at secondary school, you think that is all there is. Now I know there are lots of unsolved problems and unproved methods. And when a solution or proof has been found, a new problem will arise.

Shift away from professionals $(N=1)$ : When I started at university it was logical for me that new mathematical knowledge would develop. Now my answer is: I cannot predict the future with certainty.

Shift towards professionals $(N=6)$ : At the start of my studies I though mathematics was a more-or-less stable and complete body of knowledge. I have realised now that some theorems are only a few years old. Some of our teachers invent new mathematics. I have done a project in relatively unknown territory. There is a lot of work waiting for us. 
Table 5 (continued)

\begin{tabular}{|c|c|}
\hline Item & Explanations of shift towards and away from professionals (if any) \\
\hline 5. Unexpected solution $N=5$ & $\begin{array}{l}\text { Shift towards professionals }(N=2) \text { : At school I was convinced that by } \\
\text { thinking and estimating you could be quite sure about the kind of solutions } \\
\text { problems would bring about. Nowadays I have often seen surprising answers. } \\
\text { At school it was simply the application of rules; now a totally new argument } \\
\text { may be needed for a solution; that is what makes mathematics exciting. } \\
\text { Shift away from professionals }(N=3) \text { : The problems are more abstract and } \\
\text { more complex, so you do have fewer expectations about the solution, so } \\
\text { you cannot be surprised. }\end{array}$ \\
\hline $\begin{array}{l}\text { 17. Ready-made methods } \\
\qquad N=10\end{array}$ & $\begin{array}{l}\text { Shift towards professionals }(N=5) \text { : There are standard methods, but for } \\
\text { every case a method has to be tailored to fit the concrete problem; also it is } \\
\text { often necessary to create a convenient mathematical model. Mathematics } \\
\text { doesn't have all the answers; there are a few standard methods that can be } \\
\text { used, but the rest is theory-based improvisation. } \\
\text { Shift away from professionals }(N=5) \text { : With enough creativity and } \\
\text { manipulation of reality by problem assumptions, every technical problem } \\
\text { can be solved with mathematics. There are many standard solutions for } \\
\text { other disciplines where only the givens have to be filled in and maybe a } \\
\text { little bit remodelling. Most research and method development happens with } \\
\text { problems from within the field of mathematics. }\end{array}$ \\
\hline
\end{tabular}

\section{References}

Boaler, J. (2002a). Exploring the nature of mathematical activity: using theory, research and 'working hypotheses' to broaden conceptions of mathematics knowing. Educational Studies in Mathematics, 51, 3-21. doi:10.1023/A:1022468022549.

Boaler, J. (2002b). The development of disciplinary relationships: knowledge, practice, and identity in mathematics classrooms. For The Learning of Mathematics, 22, 42-47.

Civil, M., \& Andrade, R. (2002). Transitions between home and school mathematics: rays of hope amidst the passing clouds. In G. de Abreu, A. J. Bishop, \& N. C. Presmeg (Eds.), Transitions between contexts of mathematical practices (pp. 149-169). Dordrecht: Kluwer.

Ernest, P. (1991). The philosophy of mathematics education. UK: Routledge.

European Council. (2000). Presidency conclusions, Lisbon, 23 and 24 March 2000. Retrieved June 29, 2008, from http://cordis.europa.eu/fetch?CALLER=PROGLINK_FP6\&ACTION=D\&DOC=1\&CAT= PROG\&QUERY=1169188530042\&RCN=804.

FitzSimons, G. E. (1999). Values, vocational education and mathematics: linking research with practice. In changing practice through research: changing research through practice. Proceedings of the 7 th Annual International Conference on Post-Compulsory Education and Training (vol. 3, pp. 11-21), Centre for Learning and Work Research, Griffith University, Brisbane.

Halmos, P. (1980). The heart of mathematics. The American Mathematical Monthly, 87, 519-524. doi: $10.2307 / 2321415$.

Hoyles, C., Newman, K., \& Noss, R. (2001). Changing patterns of transition from school to university mathematics. International Journal of Mathematical Education in Science and Technology, 32, 829-845. doi:10.1080/00207390110067635.

Krogh, L. B., \& Thomsen, P. V. (2005). Studying students' attitudes towards science from a cultural perspective but with a quantitative methodology: border crossing into the physics classroom. International Journal of Science Education, 27, 281-302.

Lave, J., \& Wenger, E. (1991). Situated learning: legitimate peripheral participation. Cambridge, UK: Cambridge University Press.

National Research Council. (2001). Adding it up: helping children learn mathematics. Mathematics Learning Study Committee, Center for Education, Division of Behavioral and Social Sciences and Education. Washington, DC: National Academy Press. 
Organisation for Economic Co-operation and Development. (2006). Evolution of student interest in science and technology studies policy report. Retrieved September 29, 2008, from http://www.oecd.org/ dataoecd/16/30/36645825.pdf.

Pelgrum, W. J., Eggen, T. J. H. M., Plomp, T., \& Kuper, J. (1984). Tweede wiskunde project [in Dutch: second mathematics project]; IEA rapport. Enschede: University of Twente.

Perrenet, J., \& Adan, I. (2002). From mathematical modelling to design based learning: a bridge too far. International Journal of Mathematical Education in Science and Technology, 33, 287-297. doi:10.1080/ 00207390110097579.

Perrenet, J., \& ter Morsche, H. (2004). Modelling as a foundation for academic reflection in the mathematics curriculum. In H. W. Henn \& W. Blum (eds.), ICMI Study 14: applications and modelling in mathematics education (pp. 211-216), 13-17 February, Dortmund.

Ratner, C. (2000). Theory and method: outline of a coherent, comprehensive concept of culture. Crosscultural psychology bulletin, 34, 5-11.

Schoenfeld, A. H. (1985). Mathematical problem solving. Orlando: Academic.

Schoenfeld, A. H. (1992). Learning to think mathematically: problem solving, metacognition, and sense making in mathematics. In D. A. Grouws (Ed.), Handbook of research on mathematics teaching and learning (pp. 334-370). New York: Macmillan.

Skovsmose, O., \& Nielsen, L. (1996). Critical mathematics education. In A. J. Bishop, K. Clements, C. Keitel, J. Kilpatrick, \& C. Laborde (Eds.), International handbook of mathematics education (pp. 1257-1288). Dordrecht: Kluwer.

Vygotsky, L. S. (1978). Mind in society: the development of higher psychological processes. Cambridge, MA: Harvard University Press.

Wenger, E. (1999). Communities of practice: learning, meaning, and identity. Cambridge, UK: Cambridge University Press. 